\title{
Editorial
}

\section{The In-Hospital Consultation Today}

Whom Does It Best Serve?

Herbert L. Fred, MD, MACP

$\mathbf{W}$ hen I entered medical school 64 years ago, the primary purpose of an inhospital consultation was to gain new information, get a different perspective, or request a procedure that the referring physician was not qualified to perform. Accordingly, physicians chose their consultants carefully, contacted them personally, and rarely used go-betweens, such as nurses or ward clerks. The entire process was formal and professional.

The referring physician first sought permission for the consultation from the patient or the patient's family and later introduced the consultant at the bedside. After the consultant had examined the patient, the 2 physicians retired to a separate room to discuss the case in depth and to devise an optimal management plan for the patient. Together, they returned to the patient's bedside to explain their findings and recommendations. Consultants never wrote orders for patients unless specifically asked to do so by the referring physician. They wrote their opinions and suggestions in the progress notes or on special consultation sheets in the patient's hospital record. No matter how many consultants were involved, the referring (primary) physician always remained in charge.

That state of affairs bears little resemblance to the way that in-hospital consultations unfold today. Some of the factors responsible for the difference include the accelerated growth of medical knowledge, the widespread availability of highly advanced diagnostic and therapeutic techniques, payment schemes that require increasing numbers of patients to be seen in decreasing amounts of time, the weight of federal regulations, and the constant threat of litigation. The chief reason for the change, however, is the culture of our society. Many norms of yesteryear have essentially disappeared-especially the norm of excellence. Most people, including many physicians, are satisfied with mediocrity, the only norm they know.

Nowadays, the in-hospital consultation all too frequently serves as a convenient way for busy practitioners to save time and thought, to protect themselves from malpractice suits, or to repay colleagues for favors. Largely lost are the careful planning, formality, and professionalism that once characterized this important aspect of patient care. Moreover, contacting the consultant has become indirect and impersonal: nurses or ward clerks ordinarily function as messengers. At times, the consultant who responds is the consultant who happens to be on call, not one preferred by (or even known to) the referring physician. Commonly, the consultant's only link with the referring physician is a laboratory report, a difficult-to-decipher scribble on the paper hospital record, or one or two lines on the electronic record. Sometimes, the consultant requires that certain tests or procedures be completed before he or she actually sees the patient. Even worse, the consultant occasionally doesn't see the patient at all and renders an opinion by telephone.

One other point deserves emphasis. The referring physician rarely takes issue with the consultant, because the consultant (as an expert) "must be right" or because disagreement would be politically unwise or socially uncomfortable. Consequently, intellectual discourse rarely ensues. The consultant and any additional consultants take over the patient's care, each concentrating on a part but disregarding the whole. Meanwhile, the referring physician acts solely as an observer or triage agent. Everyone-and no one-is in charge.

So I ask: whom does this current in-hospital consultative practice best serve? The patient or ourselves? 